\title{
Chinese and Japanese Participation in B/Be Star Campaigns $^{1}$
}

\section{J. $\mathrm{HaO}^{2}$}

Beijing Astronomical Observatory, Chinese Academy of Sciences, A20 Datun Road, Chaoyang District, Beijing 100012, China National Astronomical Observatories, Chinese Academy of Sciences

\begin{abstract}
In the early 1980's, astronomers at the Beijing Astronomical Observatory (BAO) entered a collaboration in a long term photometric observing project on Be stars which included about 30 stars. For example, in 1983 the group at BAO organized a multi-site campaign to measure the short term variability of EW Lac, KX And, KY And, $o$ And, and LQ And. In 1989, 48 Per was selected to be one of the targets of MuSiCoS campaign in which many stellar astronomers at the BAO participated. In 1991, the $\mathrm{Be}$ group at the $\mathrm{BAO}$ joined a campaign on the prototypical nonradially pulsating B star, 53 Per. In 1992, French \& Chinese astronomers proposed a joint campaign on $o$ And and EW Lac. Astronomers in Japan are also active in Be star campaigns and in 1993-5 they organized several campaigns on few well known Be stars ( $\zeta$ Oph, EW Lac, $\gamma$ Cas, $\lambda$ Eri and $28 \mathrm{Cyg}$ ).
\end{abstract}

\section{International long term campaign on Be stars}

Beginning in 1983, the Be star group at Beijing Astronomical Observatory (L. Huang, Z. Gao, \& H. Jao) participated in a long term project, the International Photometric Observing Campaign of Bright Northern Be Stars organized by $\mathrm{P}$. Harmanec et al. (1981). A number of astronomers, including J. Percy (David Dunlap Obsy), L. Huang (Beijing Astronomical Observatory) and K. Pavlovski (Hvar Obsy) and their colleagues participated in this campaign. A $60 \mathrm{~cm}$ telescope equipped with single-channel electrophotometer at Xinglong Station of $\mathrm{BAO}$ was utilized to monitor stars in the UBV system. The observing facility at Xinglong Station is located on a hill of 900 meter high which is $170 \mathrm{~km}$ northwest to Beijing. The project continued during the ten year interval 1983-1992 and was conducted during the months of September-March. The reduction of the data was done with an computer code named HEC22 (written by P. Harmanec). Details of the observations can be found in many papers by these participants.

\footnotetext{
${ }^{1}$ supported by a grant of this colloquium and the Natural Science Foundation of China

${ }^{2}$ Visiting Astronomer, Beijing Astrophysics Center
} 
Our cooperative program concentrated on the long term light behavior of Be stars. Altogether it included over 30 bright northern Be stars. Not all of our results are published yet, but here are some highlights of our program:

Table 1. Targets for the International Photometric Observing Campaign of Bright Northern Be Stars

\begin{tabular}{rrrr}
\hline \hline HR 1113 & o And & EW Lac & KX And \\
KY And & LQ And & PV And & HR 7983 \\
V 1661 Cyg & HR 8020 & V832 Cyg & V 1931 Cyg \\
HR 8103 & X Per & MWC 393 & o Per \\
13 Tau & BU Tau & 17 Tau & V971 Tau \\
$\eta$ Tau & V960 Tau & $\zeta$ Tau & V731 Tau \\
$\lambda$ Eri & $\kappa$ Dra & 8 Dra & HDE 245770 \\
\hline \hline
\end{tabular}

- KX And, KY And, LQ And, o And and EW Lac (1983 Campaign, Stagg 1986)

This was a short-term mini-campaign coordinated by the participants of the long term project. Telescopes in Hvar Observatory, Kitt Peak Observatory, McDonald Observatory, David Dunlap Observatory and Xinglong Station of Beijing Astronomical Observatory were involved. Observers were K. Pavlovski, H. Bozic, J.R. Percy, L. Huang, A. Fullerton, F. Schmidt, W.S. Gao and Z.H. Guo. The results are listed in Table 2.

- A $0535+26 / \mathrm{HDE} 245770$ (Hao et al. 1996)

This X-ray system is one of the targets monitored at the Xinglong Station. We studied the 1985-92 data together with those in the literature and found a secular variation of the star over the last ten years (see Fig.1). Periods of 506d and 755d were also reported.

Table 2. Result of the 1983 Campaign on KX And, KY And, LQ And, $o$ And and EW Lac

\begin{tabular}{llll}
\hline \hline Target & $\begin{array}{l}m_{b} \\
(\mathrm{mag})\end{array}$ & $\begin{array}{l}\text { Period } \\
\text { (day) }\end{array}$ & $\begin{array}{l}\text { Amplitude } \\
\text { (mag) }\end{array}$ \\
\hline o And & 3.6 & 1.57 & 0.10 \\
KX And & 5.1 & 0.47 & 0.10 \\
KY And & 6.5 & 1.51 & 0.12 \\
LQ And & 6.7 & 0.31 & 0.03 \\
EW Lac & 6.9 & 0.72 & 0.13 \\
\hline \hline
\end{tabular}

\section{MuSiCoS Project on Be stars}

A total of $40 \mathrm{~B} / \mathrm{Be}$ stars were observed in MuSiCoS (Multi SIte COntinuous Spectroscopy) campaign during 1989-98. $48 \mathrm{Per}$ was prominent studied in the 1989 campaign. Others have recently been added to the program list. 


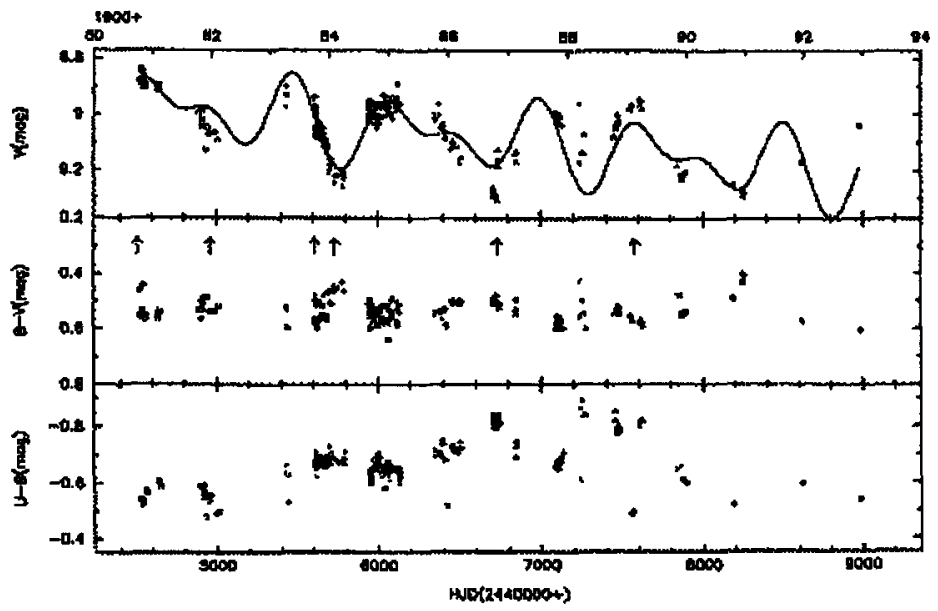

Figure 1. The photometric history of $\mathrm{HDE} 245770$ for the last decade. The solid line is the fit curve of two sinusoidal solution with periods of about $756 \mathrm{~d}\left(0.484 \mathrm{c} \mathrm{yr}^{-1}\right)$ and $\left.506 \mathrm{~d}\left(0.722 \mathrm{c} \mathrm{yr}^{-1}\right)\right)$. The small arrows at the bottom of the upper panel mark the X-ray events occurred during this period (from Hao et al. 1996).

The goal of the MuSiCoS project is to facilitate multi-site, multi-wavelength and mostly multi-program observations in stellar spectroscopy. It is shown that this type of observations is definitely needed for many scientific programs, in particular those related to stellar magnetic activities, stellar winds and stellar oscillations. Most of these programs have similar instrumental needs, namely a world network of spectroscopic facilities providing a resolving power above 30000 on telescopes of $2 \mathrm{~m}$ class. This MuSiCoS project was initially proposed by Claude Catala and Bernard Foing. The basic strategy is to gather the efforts of several groups interested in different scientific programs, all of them needing multi-site spectroscopic observations. The campaigns were multi-site, multi-program program. Each scientific program was coordinated by a principal investigator who was responsible for the scientific choices concerning the targets and observing strategy. After the campaign, the PI received raw data from participating observers. The PI was then responsible for reducing the data in a homogeneous way and organized data analysis with interested participants, and is finally responsible for publication of the results. BAO has been an active participating institution of MuSiCoS project in the last ten years. BAO astronomers were involved in almost all the MuSiCoS campaigns except for the southern one of 1994. A brief summary of the MuSiCoS project for the last decade is listed in Table 3 and 4.

48 Per is the only Be star for which results have been published (Hubert et al. 1997). This object was proposed by A. M. Hubert in the first campaign. Although there was some difficulty in unifying the data obtained from different 
Table 3. MuSiCoS workshops

\begin{tabular}{lll}
\hline \hline Date & Place & Proceedings \\
\hline 1988 & Meudon, France & yes \\
1990 & Meudon, France & no \\
1993 & Noordwijk, The Netherlands & no \\
1994 & Beijing, China & yes \\
1995 & St. Andrews, Scotland & no \\
1997 & Munich, Germany & yes* \\
\hline \hline
\end{tabular}

*Note: together with the ESO workshop on stellar wind variabilities, eds. L. Kaper and A.W. Fullerton, 1998

Table 4. Targets for $\mathrm{MuSiCoS}$ campaigns

\begin{tabular}{ll}
\hline \hline year & target \\
\hline 1989 & HR 1099, 48 Per, AB Aur \\
1992 & HR 1099, AB Aur, $\theta^{2}$ Tau \\
1994 & AB Dor, $\gamma$ Dor, $\beta$ Pic \\
1996 & AB Aur, SU Aur, V480 Tau \\
1998 & $\epsilon$ Per, $\psi^{2}$ Ori, QZ Pup, $\omega$ Ori, EI Eri, HR1099, Vela X1 \\
\hline \hline
\end{tabular}

sites for this object, efforts were made to detect the rapid spectroscopic variation by analyzing the He I $\lambda 6678$ line series. Three frequencies having values 6.04 , 0.85 and $2.77 \mathrm{c} \mathrm{d}^{-1}$ was determined. A frequency of $6.04 \mathrm{c} \mathrm{d}^{-1}$ with amplitude of $0.3 \%$ of the continuum is associated with the mode $l=10 \pm 2$.

\section{Other $\mathrm{B} / \mathrm{Be}$ campaigns}

Over last 20 years Chinese and Japanese astronomers have participated in many international campaigns on various kinds of variable stars, especially $\mathrm{B}$ and $\mathrm{Be}$ stars. As one component of our activity, we have several groups active in the $\mathrm{Be}$ star field who initiated many $\mathrm{B} / \mathrm{Be}$ campaigns themselves. In addition, due to the important geographical position in international continuous observing, Asian participation is greatly needed. These campaigns contributed to the study of the behaviors of temporal variations of $\mathrm{B} / \mathrm{Be}$ stars. Table 5 lists the campaigns to which Chinese and Japanese participants contributed.

\subsection{Campaigns with Chinese participation}

- ET And (Weiss et al. 1998):

ET And $\left(T_{\text {eff }}=12000 \mathrm{~K} \log g=3.65-3.90\right)$ is a binary system with a $\mathrm{B} 9 \mathrm{p}(\mathrm{Si})$ star as the main component. The authors reported on the photometric observing campaigns for 1988, 1989 and 1994. Their work confirmed the rotation period of $1 .{ }^{d} 618875$. Furthermore, the controversial issue of pulsational stability of ET And has been resolved since pulsation was dis- 
Table 5. B/Be campaigns with Asian participation

\begin{tabular}{|c|c|c|c|}
\hline target & epoch & type & site \\
\hline $\begin{array}{l}\text { ET And } \\
53 \text { Per } \\
\text { o And } \\
\text { EW Lac } \\
\zeta \text { Oph }\end{array}$ & $\begin{array}{l}1989 \text { Oct. } \\
1991 \text { Jan. } \\
1992 \text { Sep. } \\
1993 \text { May }\end{array}$ & $\begin{array}{l}\text { pe } \\
\text { pe,sp } \\
\text { pe,sp }\end{array}$ & $\begin{array}{l}\text { Xinglong, San Pedro, Lowell, Skalnate } \\
\text { Xinglong, Braeside, Hvar, Konkoly } \\
\text { Xinglong, Naeinonal, Braeside, San Pedro, Mt Hopkins, } \\
\text { Department of Physics, Citadal, Charleston } \\
\text { Okayama, Crimea, ESO/CAT, Dominion }\end{array}$ \\
\hline & & pe & Dodaira, ESO $/ 50 \mathrm{~cm}$ \\
\hline EW Lac & 1993 Sep. & sp & $\begin{array}{l}\text { Okayama, Crimea, Rohzenn, Ondrejov, } \\
\text { Haute Provence, Kitt Peak, Dominion }\end{array}$ \\
\hline & & pe & $\begin{array}{l}\text { Xinglong, Crimea, Belogradchik, Skalnate Pleso, } \\
\text { David Dunlap }\end{array}$ \\
\hline$\gamma$ Cas & 1993 Oct. & $\begin{array}{l}\text { pol } \\
\text { sp } \\
\text { pe } \\
\text { pol }\end{array}$ & $\begin{array}{l}\text { Okayama Crimea, Ondrejov,Haute Provence, Dominion } \\
\text { Dodaira, Xinglong, Rozhen,Alicante } \\
\text { Dominion (spectro-polarimetry) }\end{array}$ \\
\hline$\lambda$ Eri & 1994 Feb. & sp & $\begin{array}{l}\text { Okayama, Yunnan, Crimea, Ondrejov, Haute Provence, } \\
\text { Brazil, Dominion }\end{array}$ \\
\hline$\lambda$ Eri & 1994 Nov. & $\begin{array}{l}\text { pe } \\
\text { sp }\end{array}$ & $\begin{array}{l}\text { Xinglong, Alicante, Mt.Hopkins } \\
\text { Okayama, Yunnan, Ondrejov, Haute Provence, Brazil, } \\
\text { Atlanta, ESO/CAT }\end{array}$ \\
\hline & & pe & Dodaira, Xinglong, Alicante, Brazil, David Dunlap \\
\hline
\end{tabular}

covered for HD 219891 instead, which was the main comparison star. The frequency of $10.0816 \mathrm{cy} \mathrm{d}^{-1}$, a semi-amplitude of $2.5 \mathrm{mmag}, T_{\text {eff }}$ and $\mathrm{M}_{v}$ suggest that this comparison star to be a $\delta$ Scuti variable close to the blue border of the instability strip. The pulsational stability of ET And could be clearly established and hence no need exists to derive new driving mechanisms for stars between the classical instability strip and the region of slowly pulsating B-type (SPB) stars.

This study is based on total of 5 runs of observing campaigns during 5 years (1988 Oct. to 1994 Sep.). The Be group in BAO was involved in the campaign of 1988 and obtained a dataset two-weeks long.

- 53 Per (Huang et al. 1994)

The campaign was carried out in 1991 January 1-15 at four observatories on three continents (Xinglong of BAO, Braeside, Hvar, Konkoly). Frequency analysis showed that the light variations of 53 Persei during the campaign can be fitted by two sinusoids of frequencies 0.462 and 0.603 c $d^{-1}$ (see Table 6) which are practically identical to those found by others from 1977-1983 photometric and spectroscopic data. The detection of the same frequencies in the data obtained more than $10 \mathrm{yr}$ apart has convincingly confirmed the stable multiperiodicity in 53 Persei and extended the stability duration from $5.5 \mathrm{yr}$ reported in an earlier investigation to about $13 \mathrm{yr}$. This pivotal result strongly supports the nonradial pulsation as the physical cause responsible for light and line-profile variations in this prototype star. A separate analysis using both optical data from this campaign 
and UV data from the Voyager 2 satellite suggested that the active modes have a value $\ell=2$ (Smith \& Huang 1994).

Table 6. The 2-frequency fits for all data of 53 Per obtained in 1991 campaign (taken from Huang et al. 1994).

\begin{tabular}{|c|c|c|c|c|}
\hline Data & $\begin{array}{c}\text { Amplitude } \\
\text { (mag) }\end{array}$ & $\begin{array}{c}\text { Zero-point } \\
\text { (mag) }\end{array}$ & $\begin{array}{c}\text { Residuals } \\
\text { (mag) }\end{array}$ & Remarks \\
\hline$\overline{\bar{V}}$ & $\begin{array}{l}0.0317(6) \\
0.0122(21)\end{array}$ & $4.8447(16)$ & 0.0101 & \multirow{5}{*}{$\begin{array}{c}\text { Mean frequencies fixed } i\left(\mathrm{~cd}^{-1}\right): \\
f_{1}=0.4620(4), f_{2}=0.6030(1) \\
\text { Mean phases fixed (cycles): } \\
1=0.233(26), 2=0.665(11) \\
\text { Mean periods (days): } \\
P_{2}=2.1645(18), P_{2}=1.6584(3) \\
\text { Epoch of light maximum: } \\
T_{1, \max }=H J D 2448261.7244 \\
T_{2, \max }=H J D 2448261.5006\end{array}$} \\
\hline$B$ & $\begin{array}{l}0.0363(22) \\
0.0145(11)\end{array}$ & $4.8074(7)$ & 0.0108 & \\
\hline$U$ & $\begin{array}{l}0.0544(14) \\
0.0205(20)\end{array}$ & $4.2376(73)$ & 0.0216 & \\
\hline$B-V$ & $\begin{array}{l}0.0046(6) \\
0.0021(3)\end{array}$ & $-0.0374(10)$ & 0.0068 & \\
\hline$U-B$ & $\begin{array}{l}0.0179(19) \\
0.0058(5)\end{array}$ & $-0.5697(80)$ & 0.0021 & \\
\hline
\end{tabular}

- $o$ And (Sareyan et al. 1998). Huang and Sarayan teamed up to observe the stars EW Lac and $o$ And in 1992. The campaign on $o$ And lasted month. A total of 6 observing sites contributed 247 hours of photometric data and additional spectroscopic data. The light curve of $o$ And can be interpreted with the following aspects:

1. a double wave periodic curve accounts for most of the light variations,

2. secondary periods completely changed their relative importance within a few weeks.

From these results, Sareyan et al (1998) proposed a model of either rotation modulation or pulsation to interpret the behavior of $o$ And.

- EW Lac (1992 campaign) (Cao et al., in preparation)

The above campaign also included EW Lac, also over a month, and this time including four observatories. This star is characterized by both complicated long and short term variability and is a frequent target of multisite campaigns. Frequency analysis showed that periods of 9.5, 11.0 and 15.0 $\mathrm{c} \mathrm{d}^{-1}$ seemed to be the solution of the light curves plotted in Fig.2.

\subsection{Campaigns with Japanese participation}

- $\zeta$ Oph (Kambe et al. 1997)

A simultaneous multisite high-resolution spectroscopic and photometric campaign on $\zeta$ Oph (HD 149757) was made in 1993 May. A Japanese group using the $1.88 \mathrm{~m}$ telescope of the Okayama Observatory obtained 100 hours of time series observations of the He I $\lambda 6678$ line profile on successive nights. The results of this campaign showed that the period with the largest ampitude has a value of 2.018 hours $\left(f_{1}\right)$, which differs 

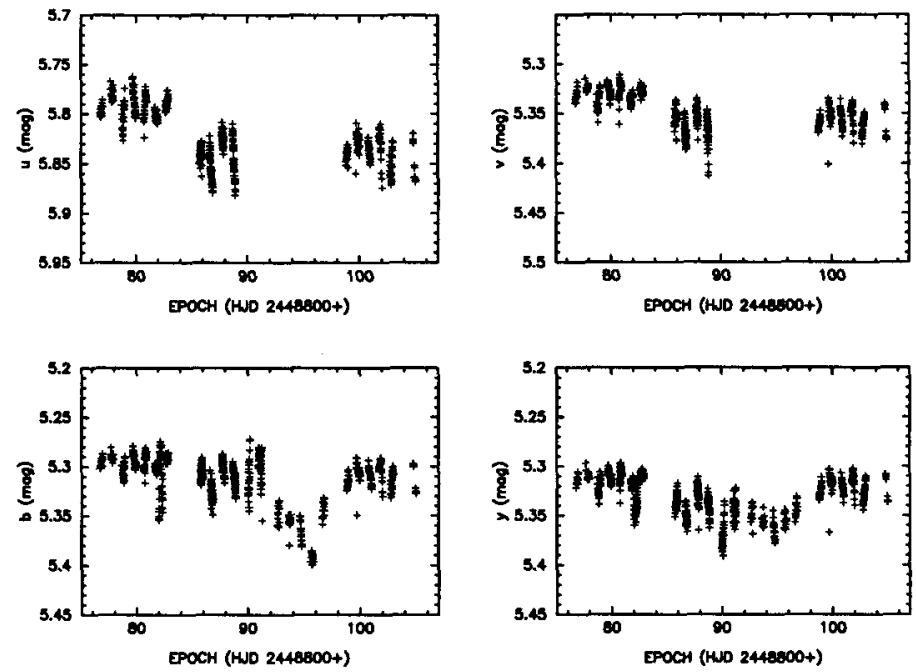

Figure 2. Light curves of EW Lac obtained in the 1992 campaign

from the 2.43 hours from previous studies. A secondary period has a value of 3.337 hours $\left(f_{2}\right)$, which is in agreement with previous studies. Periods having smaller amplitudes were found at $2.432\left(f_{3}\right), 1.257\left(f_{1}+f_{2}\right), 1.008$ $\left(2 f_{1}\right), 2.107\left(f_{4}\right), 1.293\left(f_{2}+f_{4}\right)$, and 1.668 hours $\left(2 f_{2}\right)$. Simultaneous photometric observation sshowed no counterpart periods of $f_{1}=2.018$ and $f_{2}=3.337$ hours. The authors found that the two principal periods have an approximate commensurability of 3:5, which indicates close superperiods of 10.05 hours The $l p v$ analysis leads to the following mode identification: $f_{1}$ to $l=-7$ or -8 and $f_{2}$ to $l=-4$ or -5 .

- EW Lac (1993 Campaign) (M. Floquet et al., this proceedings).

1. High $\mathrm{S} / \mathrm{N}$ spectra of the $\mathrm{He} \mathrm{I} \lambda 6678$ and $\mathrm{H} \alpha$ lines, and polarization data were obtained in 1993 August-September.

2. All quantities derived (equivalent width, RV, FWHM, V and R emission components) and line profiles of HeI6678 show clearly the superposition of mid-term and short-term variations.

- $\lambda$ Eri (E. Kambe et al., these proceedings)

1. a tesseral toroidal mode (e.g. $l=5, m=2$ ) can reproduce the observed $l p v$ with the 16.9 hour period well,

2. the far more complicated $l p v$ with the $6.45 \mathrm{hr}$ period can also be explained by toroidal modes with intermediate $m$ (e.g., $l=9, m=5$ ),

3 . temperature variations due to NRP were discussed.

Acknowledgments. The author thanks Drs. Hirata and Kambe for necessary references for the preparation of this talk. A grant by the colloquium organizers which permitted me to attend the meeting is acknowledged. 


\section{References}

Hao, J., Huang, L., \& Guo, Z. 1996, A\&A 308,499

Harmanec, P., Horn, J., \& Koubsky, P. 1981, Be star Newsletter, 2, 3

Hirata, R. 1994, IAU Symp. 163, ed. L. Balona, H. Henrichs, \& J. LeContel, (Kluwer: Dordrecht),

Huang, L. 1986, in the Second Japan-China Workshop on Stellar Activity and

Observational Techniques, review talk (unpub.)

Huang, L., Guo, Z., Hao, J., Percy, J. R., Fieldus, M. S., Fried, R., Pavlovski, K., Bozic, H., Ruzic, Z., Paparo, M., \& Veto, B. 1994, ApJ 431, 850

Hubert, A. M., Floquet, M., Hao, J., Caillet, S., Catala, C., Foing, B. H., Neff, J.E., Huang, L., Hubert, H., Barban, C., Baudrand, J., Cao, H., Char, S., Chatzichristou, H., Cuby, J. G., Czarny, J., Dreux, M., Felenbok, P.,Guérin, J., Hron, J., Huovelin, J., Jankov, S., Jiang, S., Le Contel, J.M., Maitzen, H.M., Petrov, P., Savanov, I., Shcherbakov, A., Simon, T., Stee, P., Tuominen, I., \& Zhai, D. 1997, A\&A 324, 929

Kambe, E., Hirata, R., Ando, H., Cuypers, J., Katoh, M, Kennelly, E.J., Walker, G.A.H., Štefl, S.,\& Tarasov, A. E. 1997, ApJ 481, 406

Sareyan, J. P.,Gonzalez-Bedolla, S., Guerrero, G., Chauville, J., Huang, L., Hao, J., Guo, Z., Adelman, S. J., Briot, D., Alvarez, M. 1998, A\&A 332, 155

Smith, M. A., \& Huang, L. 1994, IAU Symp. No. 163, op. cit., p.37

Stagg, C. R. 1986, unpub. Ph.D Thesis, University of Toronto

Weiss, W. W., Kuschnig, R., Mkrtichian, D. E., Kusakin, A. V.,Kreidl, T. J., Bus, S. J., Osip, D. J., Guo, Z., Hao, J., Huang, L., Sareyan, J. P., Alvarez, M., Bedolla, S.G., Zverko, J.,Žižñovský, J., Mittermayer, P., Zwintz, K., Polosukhina, N., Mironov, A.V., Dorokhov, N. I., Goranskij V. P., Dorokhova, T. N., Schneider, H., Heisberger, F. 1998, A\&A 338, 919

\section{Discussion}

N. Ashok: A program to study variability of Be stars has been initiated at Physical Research Laboratory, Ahmedabad, India. Near-IR and optical spectra with resolution of 1,000 and 10,000 , respectively, will be obtained with the $1.2 \mathrm{~m}$ telescope of Mt. Abu Observatory. The geographical location $\left(70^{\circ} 46^{\prime} 48^{\prime \prime} \mathrm{E}\right.$, $24^{\circ} 39^{\prime} 9^{\prime \prime} \mathrm{N}$ ) nicely bridges the gap between Chinese and European observatories resulting in better temporal coverage. During the period January to April 1999 near-infrared and optical spectra of about $40 \mathrm{Be}$ stars have been obtained. In the coming years participation in international campaigns will be started. 